\title{
Correlations between Antioxidant Activity and Alkaloids and Phenols of Maca (Lepidium meyenii)
}

\author{
Jin Gan, Ying Feng, Zhao He, Xian Li, and Hong Zhang \\ Research Institute of Resource Insects, Chinese Academy of Forestry, Bailongsi, Kunming, Yunnan 650224, China \\ Correspondence should be addressed to Jin Gan; ganjin638@126.com
}

Received 28 April 2017; Revised 5 September 2017; Accepted 16 September 2017; Published 15 October 2017

Academic Editor: Ángel A. Carbonell-Barrachina

Copyright (C) 2017 Jin Gan et al. This is an open access article distributed under the Creative Commons Attribution License, which permits unrestricted use, distribution, and reproduction in any medium, provided the original work is properly cited.

\begin{abstract}
The antioxidant capacity of maca has been considered to be the basis for other bioactivities, and revealing the active antioxidant compounds would help to elucidate a variety of bioactive compounds. In this study, the correlation between the antioxidant activity of maca and secondary metabolites, including ferric reducing antioxidant potential (FRAP), hydroxyl radical scavenging ability (HRSA), lipid peroxidation inhibition ability (LPIA), total phenolic contents (TPCs), total alkaloid contents (TACs), and total sterol contents (TSCs), was investigated by measuring. Chloroform was selected to be an efficient extraction solvent for antioxidant compounds in maca by polarity fractions test. The results showed that TPC exhibited significant linear correlations $(P<0.05)$ to FRAP and LPIA, while TAC had significant linear correlations $(P<0.05)$ to FRAP, HRSA, and LPIA. These results suggested that alkaloids and phenols were the most important substances for the antioxidation of maca, of which the antioxidant effect of alkaloids seemed to be higher than that of phenols.
\end{abstract}

\section{Introduction}

Maca (Lepidium meyenii) is a plant in the Brassicaceae family that grows at an altitude of approximately 4000 to 4500 meters in the Andean region of Peru. The underground part of the plant, tuberous root, is the main product of maca. The cultivation of maca has been found to date back as far as the 16th century [1]. Maca has been traditionally used for food and to increase nutrition and human fertility. In recent years, maca has gained significant interest due to its variety of bioactivities, such as fertility-enhancing properties $[2,3]$, sexual function improvement $[4]$, antifatigue $[5,6]$, increasing immunity function [7], inhibition of prostate hyperplasia [8], and reducing menopause syndrome [9] that have been demonstrated by previous studies.

The antioxidation of maca in vitro and in vivo has been confirmed by several research studies. From an in vitro method, the research of Sandoval et al. [10] showed that aqueous extracts of maca have the capacity to scavenge free radicals and protect cells against oxidative stress. In a study of the in vitro antioxidant activity of ethanol extracts of maca cultivated in Yunnan, ethanol extracts from 3 colored types (white, yellow, and purple) of maca were shown to have dose-dependent total antioxidant and free radical scavenging effects, as measured by the ferric reducing antioxidant potential (FRAP), hydroxyl radical scavenging ability (HRSA), and 2,2-diphenyl-1-picrylhydrazyl (DPPH) free radical scavenging capacity [11]. In vivo, Večeřa et al. [12] discovered that the activity of SOD (superoxide dismutase) in the liver, GSH-Px (glutathione peroxidase) in the blood, and the level of GSH (glutathione) in the liver were all significantly increased after rats were fed fodder containing $1 \%$ maca for 2 weeks. Mice from a natural aging model were orally given ethanol extracts of maca for $60 \mathrm{~d}$, and the activity of GSH-Px in the liver and heart as well as SOD in the sera and liver increased, while MDA (malondialdehyde) in the sera and liver decreased, suggesting that ethanol extracts of maca possess antisenility effects by improving free radical metabolism [13]. Both maca stock and its ethanol extract have demonstrated antioxidant activity. However, a number of researchers have studied a variety of maca bioactivities, including improved growth rate [14], reduced menopause syndrome [9], antiproliferative functions [15], increased swimming endurance capacity [16], and enhanced sperm quantity and quality $[17,18]$. These bioactivities have 
been hypothesized as a result of antioxidation properties of maca, which are based on the capacity of scavenging free radicals, inhibition of lipid peroxidation, improved activity of antioxidant enzymes, and attenuation of oxidative stress.

Based on the previously mentioned qualities of maca, the antioxidant activity of maca seems to be highly correlated with its other bioactivities; thus, the results of studies on the antioxidant activity of maca compounds would be valuable to discover a variety of bioactive compounds. Maca contains secondary metabolites, such as alkaloids (including macamides), phenols, sterols, and glucosinolates, which are regarded as its main bioactive compounds. However, to date there is a lack of information concerning the exact bioactivities of compounds that correspond to a particular function. Therefore, the objective of this study was to investigate the correlation between the antioxidant activity in vitro and secondary metabolites, including alkaloids, phenols, and sterols, using the antioxidant activity guided fractionation method to reveal the antioxidative substances of maca.

\section{Materials and Methods}

2.1. Plant Materials. Maca (yellow) plants were collected in January after seven months of growth in testing grounds belonging to the Research Institute of Resource Insects of Chinese Academy of Forestry, which is located in the Wumeng area in Yunnan province (China) at $4000 \mathrm{~m}$ above sea level. The samples (hypocotyls) were thoroughly washed with water and then stored at $4^{\circ} \mathrm{C}$ until extraction.

2.2. Chemicals. Matrine, gallic acid, $\beta$-sitosterol, and campesterol were of standard grade and were purchased from the National Institutes for Food and Drug Control (Beijing, China). Hexane was of chromatographic pure grade and was purchased from Thermo Fisher Scientific (China) Co. Ltd. (Shanghai, China). 4,6-Tripyridyl-triazine (TPTZ) was of biochemical reagent grade and was purchased from Sigma-Aldrich (Shanghai) Trading Co. Ltd. (Shanghai, China). Ethanol, petroleum ether, chloroform, ethyl acetate, and n-butanol were of analytical grade and were purchased from Kunming Senghua Chemical Reagent Co. Ltd. (Kunming, China). $\mathrm{FeSO}_{4}, \mathrm{FeCl}_{3}$, salicylic acid, butylated hydroxytoluene, linoleic acid, thiobarbituric acid, Folin-Ciocalteu reagent, and bromothymol blue were of analytical grade and were purchased from Sinopharm Chemical Reagent Co. Ltd. (Shanghai, China).

2.3. Extraction and Separation. The antioxidant activity guided fractionation method was employed. The refrigerated maca hypocotyls were cut into slices and soaked in aqueous ethanol $(70 \%, v / v)$ at room temperature for 24 hours with a solid to solvent ratio of $1: 10(\mathrm{w} / \mathrm{v})$ in triplicate, and the combined filtrate was concentrated using a rotary evaporator (Tokyo, Japan) under vacuum at $40^{\circ} \mathrm{C}$ to a constant weight to obtain the hydroalcoholic extract (HE) of maca. The extract was suspended in deionized water and partitioned using different solvents in the following order: petroleum ether, chloroform, ethyl acetate, and n-butanol. The residue was the aqueous fraction. The partitioned extracts were concentrated to a constant weight to obtain the petroleum ether (PF), chloroform (CF), ethyl acetate (EF), n-butanol (BF), and aqueous (AF) fractions and stored at $44^{\circ} \mathrm{C}$ prior to analysis of antioxidant activities and secondary metabolite contents.

The chloroform fraction was further separated using a silica gel column. Briefly, the chloroform fraction was dissolved in chloroform, silica gel was added at a ratio of $1: 1.5(\mathrm{w} / \mathrm{w})$, and the mixture was packed into the silica gel column after drying naturally. The compounds were eluted from the silica gel column in the order of increasing polarity using 6 elution solvents with different polarities (mixed with petroleum and ethyl acetate at ratios of $10: 1,5: 1,1: 1,0: 1$, as well as $100 \%$ acetone and $100 \%$ methanol) through a C620 BUCHI medium pressure system (BUCHI Laboratory Equipment Trading (Shanghai) Ltd., Shanghai, China). The corresponding eluents were concentrated under vacuum at $40^{\circ} \mathrm{C}$ to constant weights to obtain the constituents labeled $\mathrm{C1}, \mathrm{C} 2, \mathrm{C} 3, \mathrm{C} 4, \mathrm{C} 5$, and $\mathrm{C} 6$ and stored at $4^{\circ} \mathrm{C}$ prior to analysis of antioxidant activities and determination of secondary metabolite contents.

\subsection{Analysis of Antioxidant Activities}

2.4.1. Ferric Reducing Antioxidant Potential Assay (FRAP Assay). The FRAP assay was performed according to the method of Benzie and Strain [19] with slight modifications. Briefly, the FRAP reagent was freshly prepared by mixing $10 \mathrm{mM}$ 4,6-tripyridyl-triazine (TPTZ) in $40 \mathrm{mM} \mathrm{HCl}, 20 \mathrm{mM}$ $\mathrm{FeCl}_{3}$, and $30 \mathrm{mM}$ acetate buffer $(\mathrm{pH} 3.6)$ at a ratio of $1: 1: 10$ (by volume) prior to evaluation. A total of $120 \mu \mathrm{L}$ of extracts $(3.2 \mathrm{mg} / \mathrm{mL}), 80 \mu \mathrm{L}$ of distilled water, and $3.8 \mathrm{~mL}$ of FRAP reagent were transferred into a tube and incubated at $37^{\circ} \mathrm{C}$ for $10 \mathrm{~min}$. Absorbances were measured at $593 \mathrm{~nm}$ using a Thermo Varioskan Flash (Thermo Fisher Scientific, USA) relative to a reagent blank that was also incubated at $37^{\circ} \mathrm{C}$. The FRAP data for samples were analyzed according to a standard curve of $\mathrm{FeSO}_{4}(0.1-0.8 \mathrm{mmol} / \mathrm{mL})$. The results were expressed as $\mathrm{mg} \mathrm{FeSO}_{4} / \mathrm{g}$ extract. Each extract was assayed in triplicate.

\subsubsection{Hydroxyl Radical Scavenging Ability Assay (HRSA} Assay). Hydroxyl radical scavenging ability was measured according to the method of Xu and Qin [20] with some modifications. The reaction system contained $0.5 \mathrm{~mL}$ of $9 \mathrm{mM}$ FeSO4, $0.5 \mathrm{~mL}$ of $9 \mathrm{mM}$ salicylic acid-ethanol, $2.8 \mathrm{~mL}$ of deionized water, and $200 \mu \mathrm{L}$ of extracts $(3.2 \mathrm{mg} / \mathrm{mL})$. The reaction was started by adding $0.5 \mathrm{~mL}$ of $8.8 \mathrm{mM}$ hydrogen peroxide. After incubating for $30 \mathrm{~min}$ at $37^{\circ} \mathrm{C}$, the absorbance of the reaction solution was measured at $510 \mathrm{~nm}$ by a Thermo Varioskan Flash (Thermo Fisher Scientific, Waltham, Massachusetts, USA) using butylated hydroxytoluene (BHT) as a positive control. Each extract was assayed in triplicate. The hydroxyl radical scavenging ability was calculated using the following formula:

$$
\text { Scavenging ability }(\%)=\frac{A o-A i}{A o} \times 100 \% \text {, }
$$

where $A i$ was the absorbance of the extracts or BHT and Ao was the absorbance of the control group with $0.5 \mathrm{~mL}$ of deionized water instead of extracts. 
2.4.3. Lipid Peroxidation Inhibition Ability Assay (LPIA Assay). Lipid peroxidation inhibition ability was determined according to the slightly modified method of $\mathrm{Ng}$ et al. [21]. The reaction system contained $1.0 \mathrm{~mL}$ of linoleic acid emulsion, $0.2 \mathrm{~mL}$ of $\mathrm{FeSO}_{4}, 0.2 \mathrm{~mL}$ of $\mathrm{H}_{2} \mathrm{O}_{2}, 0.6 \mathrm{~mL}$ of extracts $(3.2 \mathrm{mg} / \mathrm{mL})$, and $0.4 \mathrm{~mL}$ of deionized water and was incubated at $37^{\circ} \mathrm{C}$ for $30 \mathrm{~min}$. The reaction was stopped by adding $0.05 \mathrm{~mL}$ of trichloroacetic acid (TCA, 20\%, w/w) and $2.0 \mathrm{~mL}$ of thiobarbituric acid (TBA, $0.8 \%, \mathrm{w} / \mathrm{w})$, then the solution was mixed completely and heated at $100^{\circ} \mathrm{C}$ for $30 \mathrm{~min}$. After cooling, $4 \mathrm{~mL}$ of n-butyl alcohol was added to leach the malondialdehyde- (MDA-) TBA complex, then the mixture was centrifuged at $3000 \mathrm{rpm}$ for $10 \mathrm{~min}$, and the absorbance of the supernatant was measured at $532 \mathrm{~nm}$ using a Thermo Varioskan Flash (Thermo Fisher Scientific, USA). Butylated hydroxytoluene (BHT) was used as a positive control. Each extract was assayed in triplicate. The inhibition ability (\%) was calculated using the following formula:

$$
\text { Inhibition ability }(\%)=\frac{A o-A i}{A o} \times 100 \% \text {, }
$$

where Ao was the absorbance of the control with deionized water instead of extracts and $A i$ was the absorbance of the tested extracts.

\subsection{Determination of Secondary Metabolites}

2.5.1. Determination of Total Phenolic Content (TPC). TPC was determined according to the Folin-Ciocalteu method [22] with some modifications. In brief, $0.0200-0.2000 \mathrm{~g}$ of each extract was dissolved in $25 \mathrm{~mL}$ of $70 \%(\mathrm{v} / \mathrm{v})$ ethanol, and the mixture was centrifuged at $3000 \mathrm{rpm}$ for $15 \mathrm{~min}$. The supernatant was filtered using filter paper, the residue was reextracted twice under the same conditions, and the combined filtrate was concentrated using a rotary evaporator (EYELA, Japan) under vacuum at $40^{\circ} \mathrm{C}$. The extract volumes were adjusted to $25 \mathrm{~mL}$ with $70 \%(\mathrm{v} / \mathrm{v})$ ethanol before they were stored in the dark at $4^{\circ} \mathrm{C}$ until further use. An aliquot of $0.5 \mathrm{~mL}$ of extract prepared above was mixed with $4 \mathrm{~mL}$ of deionized water and $1 \mathrm{~mL}(1 \mathrm{~mol} / \mathrm{L})$ Folin-Ciocalteu reagent. After $5 \mathrm{~min}, 3 \mathrm{~mL}$ of $10 \%(\mathrm{w} / \mathrm{w})$ sodium carbonate was added to the previous mixture and filled to $10 \mathrm{~mL}$ with deionized water. The absorbances of the reaction products were measured using a Thermo Varioskan Flash (Thermo Fisher Scientific, USA) at $765 \mathrm{~nm}$ after $2 \mathrm{~h}$ of incubation at $30^{\circ} \mathrm{C}$. The same procedure was repeated using different concentrations $(0-60 \mu \mathrm{g} / \mathrm{mL})$ of gallic acid to plot calibration curves. The total phenolic content of each extract was calculated from the gallic acid calibration curve, and the results were expressed as milligrams of gallic acid equivalents (GAE) per gram of dry weight of extract (mg GAE/g extract). Each extract was determined in triplicate.

2.5.2. Determination of Total Alkaloids Content (TAC). TAC was analyzed using the method described by Gan et al. [23] with slight modifications. An aliquot of $0.0200-0.2000 \mathrm{~g}$ of each extract was dissolved in $20 \mathrm{~mL}$ of methanol and centrifuged at $3000 \mathrm{rpm}$ for $10 \mathrm{~min}$ in triplicate, then the methanol in the combined supernatant was recovered using a vacuum rotary evaporation apparatus (EYELA, Japan) at $40^{\circ} \mathrm{C}$. The obtained extract was dissolved in $2 \% \mathrm{HCl}$ and filtered. The filtrate, which was adjusted to $\mathrm{pH} 10$ using $10 \%$ $\mathrm{NaOH}$, was extracted with the same volume of chloroform 3 times. The combined extract was concentrated, filled to $10 \mathrm{~mL}$, and used to determine total alkaloids. A total of $1 \mathrm{~mL}$ of the extract prepared above was mixed with $5 \mathrm{~mL}$ of $2.0 \times 10^{-4} \mathrm{~mol} / \mathrm{L}$ bromothymol blue chromogenic agent $(\mathrm{pH}$ 7.0) and $5 \mathrm{~mL}$ of chloroform in a $25 \mathrm{~mL}$ colorimetric tube and shaken for $2 \mathrm{~min}$. The mixture was transferred into a separating funnel and left to sit for $2 \mathrm{~h}$, then the chloroform layer located in the bottom of the funnel was collected, of which $4 \mathrm{~mL}$ was removed and $0.2 \mathrm{mg}$ of anhydrous sodium sulfate was added to the removed portion and shaken to dehydrate the solution. After sitting for $10 \mathrm{~min}$, the absorbance of the supernatant was measured at $414 \mathrm{~nm}$ using a Thermo Varioskan Flash (Thermo Fisher Scientific, USA). The TAC of the extract was calculated using a standard curve of matrine $(0.01-0.06 \mathrm{mg} / \mathrm{mL})$ and expressed as milligrams of matrine equivalents (ME) per gram of dry weight of extract (mg ME/g extract). Each extract was determined in triplicate.

2.5.3. Determination of Total Sterol Content (TSC). TSC was determined according to the method of Gan et al. [24] with slight modifications. Each extract $(0.5000-5.0000 \mathrm{~g})$ was extracted with petroleum ether using a soxhlet apparatus for $2 \mathrm{~h}$ at $80^{\circ} \mathrm{C}$ and concentrated to a constant weight to obtain the lipid components. The obtained lipid components were added to $10 \mathrm{~mL}$ of $2.5 \mathrm{~mol} / \mathrm{L}$ sodium hydroxide and shaken strongly for $30 \mathrm{~s}$. The mixture was heated for saponification in a water bath at $85^{\circ} \mathrm{C}$ for $1 \mathrm{~h}$, then $10 \mathrm{~mL}$ of a saturated sodium chloride solution and $20 \mathrm{~mL}$ of $n$-hexane were added after cooling to room temperature. The mixture was shaken strongly for $3 \mathrm{~min}$ and centrifuged at $5000 \mathrm{rpm}$ for $10 \mathrm{~min}$. The aqueous solution in the bottom of the flask was extracted with $20 \mathrm{~mL} n$-hexane 3 times, and $10 \mathrm{~g}$ of sodium sulfate was added to the combined hexane extract to dehydrate the solution. The solution was filtered with filter paper, concentrated, and filled to $10 \mathrm{~mL}$.

The hexane extract was filtered through a $0.22 \mu \mathrm{m}$ filter and injected into the Thermol ITQ900 GC-MS system (Thermo Fisher Scientific, USA). The analysis column was a TR-5MS $(30 \mathrm{~m} \times 250 \mu \mathrm{m}$ ID $\times 0.25 \mu \mathrm{m}$ film $)$ capillary column (Thermo Fisher Scientific, USA). The gas chromatographic conditions were as follows: the injection volume was $2 \mu \mathrm{L}$, split ratio was $20: 1$, inlet temperature was $330^{\circ} \mathrm{C}$, and helium carrier gas flow rate was $1.5 \mathrm{~mL} / \mathrm{min}$. The temperature was programmed as follows: initial temperature of $220^{\circ} \mathrm{C}$ was maintained for $1 \mathrm{~min}$, increased to $300^{\circ} \mathrm{C}$ at a rate of $10^{\circ} \mathrm{C} / \mathrm{min}$, and maintained for $10 \mathrm{~min}$. The mass source was EI, temperatures of the ion source and transmission line were $280^{\circ} \mathrm{C}$ and $300^{\circ} \mathrm{C}$, respectively, scanning mode was full scan, solvent delay was $3 \mathrm{~min}$, and quality of the scanning range was from 40 to 450 . Sterol compounds were identified by the NIST2.0 standard mass spectrum database retrieval and their retention times were compared with pure standards. The sterol contents were calculated according to a standard curve plotted using standard concentrations $(0.06-0.12 \mathrm{mg} / \mathrm{mL}$ of $\beta$-sitosterol and $0.04-0.08 \mathrm{mg} / \mathrm{mL}$ of 

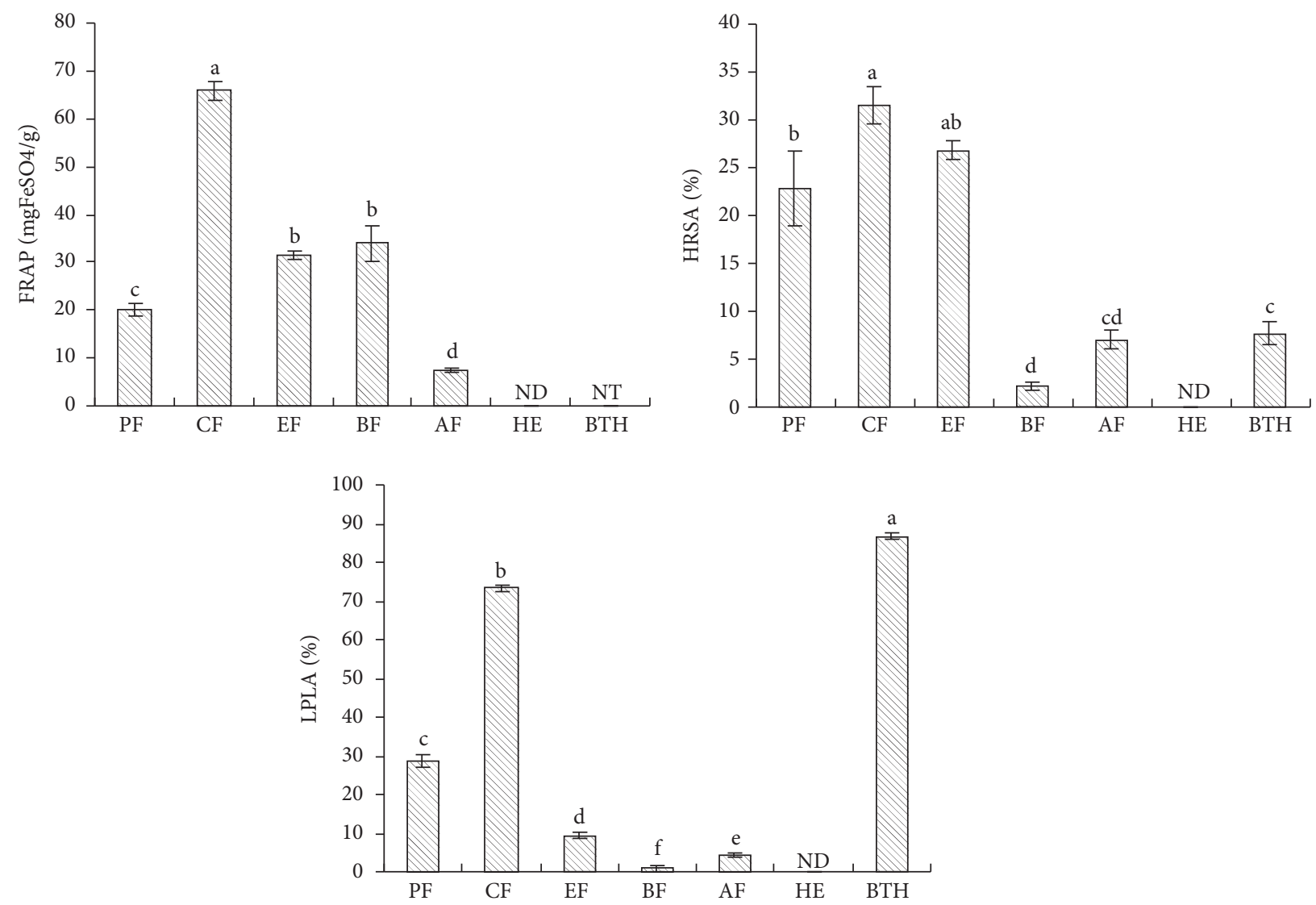

FIGURE 1: Antioxidant activities of the polarity fractions and hydroalcoholic extracts from maca. The values are means \pm standard deviations of triplicate analyses. Columns labeled with different letters are significantly different $(P<0.01)$ by Duncan test. NT means not tested. ND means not detected.

campesterol) and corresponding peak areas. TSC was equal to the sum of $\beta$-sitosterol and campesterol contents. The results were expressed as $\mathrm{mg} / 100 \mathrm{~g}$ extract of the dry weight of the extract (mg/100 g DW).

2.6. Statistical Analysis. All data were expressed as means \pm standard deviations. The differences between the means were analyzed by Duncan's test of One-Way ANOVA using SPSS 13.0 for Windows (SPSS Inc., Chicago, IL, USA). Correlations and regression analysis between the antioxidant activities of the three independent tests (FRAP, HRSA, and LPIA) and contents of secondary metabolites, including TPC, TAC, and TSC were conducted using the regression program in SPSS 13.0.

\section{Results and Discussion}

3.1. Antioxidant Activities of Polarity Fractions. Antioxidants are usually involved in several mechanisms of action, including inhibition of free radical generation, enhancement of scavenging capacity against free radicals, and reducing power; thus no single assay can accurately reflect all of the antioxidants in a mixed or complex system. Therefore, it is necessary to use at least two different methods to evaluate the antioxidant capacities of products [25]. In this study, three antioxidant assays, which were ferric reducing antioxidant potential (FRAP), hydrogen radical scavenging ability (HRSA), and lipid peroxidation inhibition ability (LPIA), were applied to evaluate the antioxidant properties of the hydroalcoholic extracts $(\mathrm{HE})$ of fresh maca and five fractions (PF, CF, EF, BF, and $\mathrm{AF}$ ). The contents of secondary metabolites, including TPC, TAC, and SC from five fractions, were also determined.

The results of the FRAP assay in Figure 1 show that values from various polarity fractions were significantly different $(P<0.01)$, and the FRAP value of $\mathrm{CF}$ was the highest $\left(65.81 \mathrm{mg} \mathrm{FeSO}_{4} / \mathrm{g}\right)$. Similarly, the hydroxyl radical scavenging abilities of five polar fractions were also significantly different $(P<0.01)$. The HRSA of CF was the highest $(31.52 \%)$, and the abilities of three fractions (CF, EF, and PF) were markedly higher than that of BHT. The analysis results of LPIA indicated that the inhibitory ability of BHT was the highest, while the inhibitory ability of CF was lower than that of BHT $(86.70 \%)$ but significantly higher $(73.26 \%)$ than those of the other fractions (0.87-28.68\%). According to the above results, CF had the highest antioxidant activity, as measured by FRAP, HRSA, and LPIA.

3.2. Contents of Secondary Metabolites of Polarity Fractions. For the assay of secondary metabolite contents (Figure 2), 

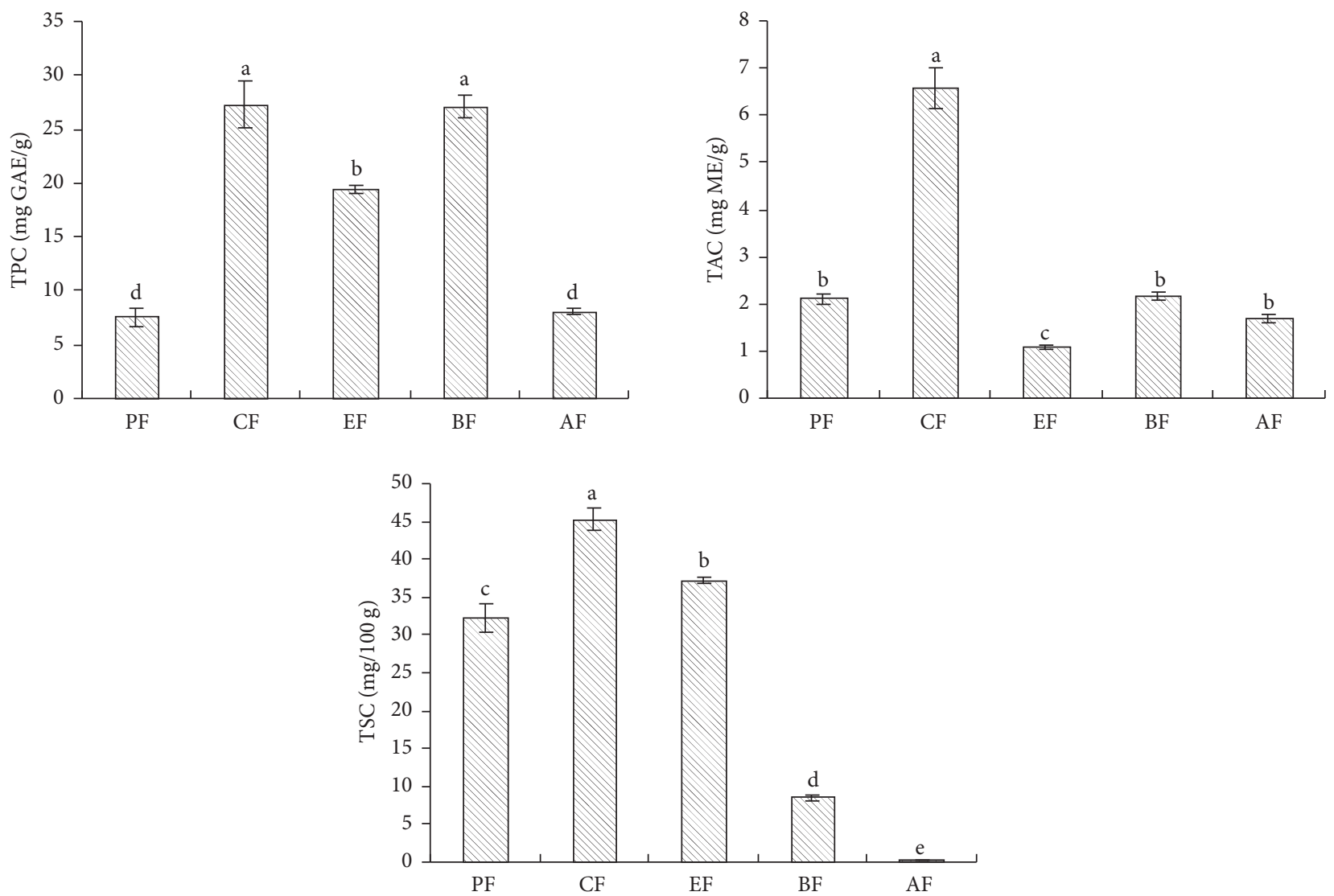

FIGURE 2: Contents of secondary metabolite of the polarity fractions and hydroalcoholic extracts from maca. The values are means \pm standard deviations of triplicate analyses. Columns labeled with different letters are significantly different $(P<0.01)$ by Duncan test.

the TPC of CF was approximately the same as BF with 27.29 and $27.05 \mathrm{mg} \mathrm{GAE} / \mathrm{g}$, respectively, and they were both significantly higher than the contents of the other fractions. The TAC of CF was $6.56 \mathrm{mg} \mathrm{ME} / \mathrm{g}$, which was significantly higher than the contents of the other four fractions, while EF had the lowest TAC. The TSCs of the five fractions ranged from 0.27 to $45.23 \mathrm{mg} \mathrm{ME} / \mathrm{g}$ and were significantly different, and the CF value was the highest. The secondary metabolite determination results indicated that CF had the highest TPC, TAC, and TSC.

No antioxidant activity was found in the hydroalcoholic extracts (HE) measured by FRAP, HRSA, and LPIA under equal concentrations with polarity fractions (Figure 1), while secondary metabolite contents, including TPC, TAC, and TSC, significantly varied between the five fractions. Moreover, some of the fractions showed an even higher antioxidant activity than that of the well-recognized synthetic antioxidant BHT, indicating that the active antioxidant compounds were separated and gathered after polarity partitioning. In this study, the chloroform fraction concurrently possessed the highest antioxidant activity and secondary metabolite contents, agreeing with reports that found chloroform to be an efficient extraction solvent for antioxidant compounds from plant materials [26, 27]. Unlike this result, the $n$ butanol extract of maca has been reported to contain high phenolic contents and total antioxidant activity, and total antioxidant activity is significantly correlated with phenolic contents [28]. However, according to our study, the TPC of the n-butanol fraction was approximately the same as the chloroform fraction, but the antioxidant activity was much lower, suggesting that the antioxidant activity of maca might be related to not only phenols but also other compounds such as alkaloids.

3.3. Antioxidant Activities of Constituents Separated from Chloroform Fraction. The chloroform fraction, which had higher antioxidant activities according to the above results, was further separated using a silica gel column to obtain various constituents labeled $\mathrm{C} 1, \mathrm{C} 2, \mathrm{C} 3, \mathrm{C} 4, \mathrm{C} 5$, and C6. The antioxidant activities (FRAP, HRSA, and LPIA) and secondary metabolite contents (TPC, TAC, and TSC) of the constituents were analyzed.

As indicated in Figure 3, the FRAPs of C4, C5, and C6 ranged from approximately 80.54 to $82.42 \mathrm{mg} \mathrm{FeSO}_{4} / \mathrm{g}$, which were much higher than those of the other constituents (15.45-29.9 $\mathrm{mg} \mathrm{FeSO}_{4} / \mathrm{g}$ ), and was significantly higher than that of CF $\left(71.15 \mathrm{mg} \mathrm{FeSO}_{4} / \mathrm{g}\right)$. The HRSA of C6 was the highest $(49.59 \%)$ for all of the constituents and CF, while other constituents were lower than CF (25.55\%). No LPIA values were detected in $\mathrm{C} 1, \mathrm{C} 2$, and $\mathrm{C} 3$. The LPIA of C6 was the highest (71.95\%), followed by $\mathrm{C} 4$ and $\mathrm{C} 5$, and CF had the lowest value of only $7.57 \%$. 

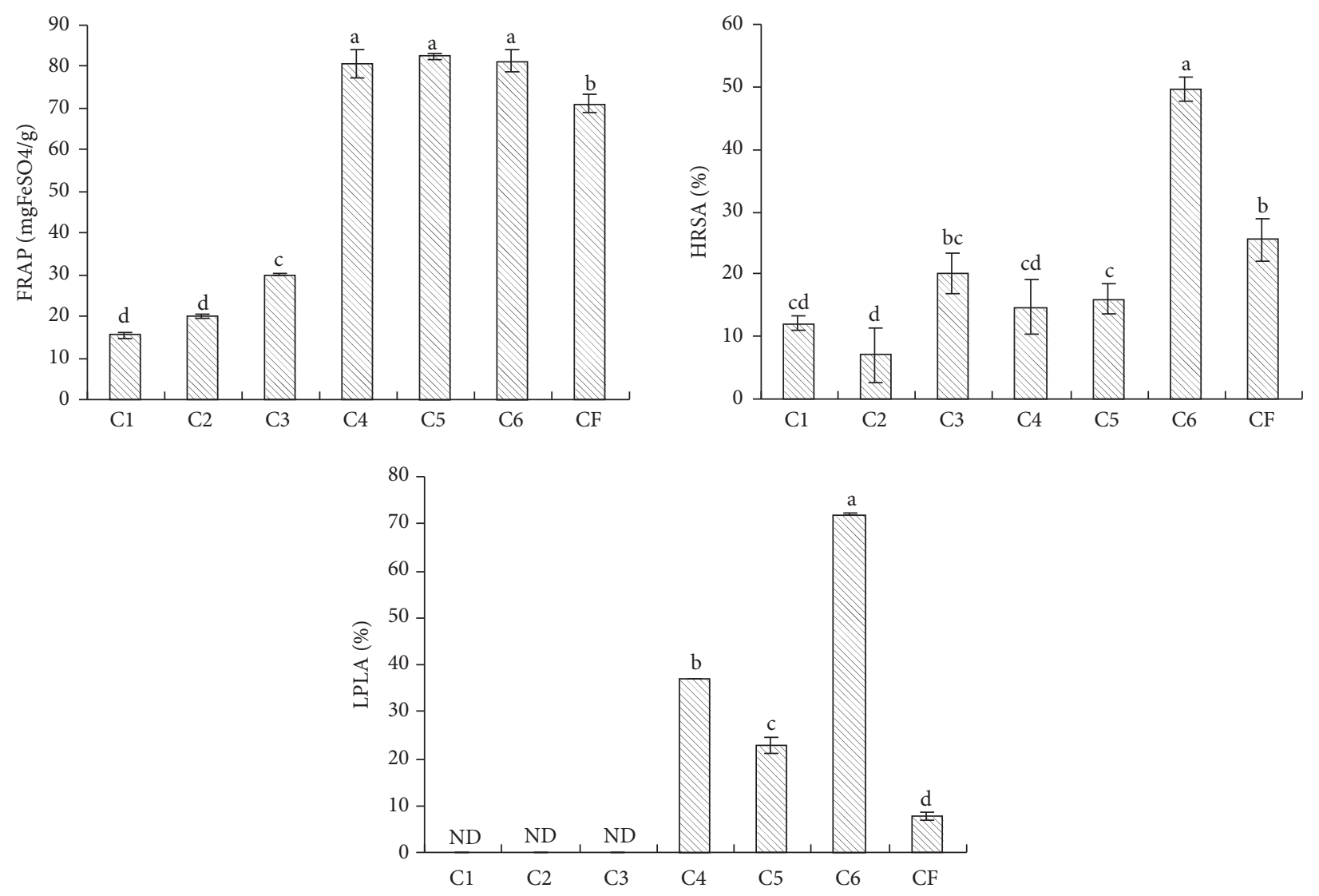

Figure 3: Antioxidant activities of constituents separated from the chloroform fraction. The values are means \pm standard deviations of triplicate analyses. Columns labeled with different letters are significantly different $(P<0.01)$ by Duncan test. ND means not detected.

3.4. Secondary Metabolite Contents of Constituents Separated from Chloroform Fraction. Figure 4 shows that the secondary metabolite contents, including TPC, TAC, and TSC of the various constituents, were significantly different $(P<0.01)$. The TPCs of C4, C5, and C6 ranged from 31.6 to $44.72 \mathrm{mg}$ GAE/g and were higher compared with the TPCs of the other constituents and CF (27.29 GAE mg/g). The TAC of C6 was not only the highest among all of the constituents but was also higher than that of CF. The TSC of C2 (171.6 mg/100 g) was much higher than those of the other constituents and CF, which ranged from 3.3 to $15.63 \mathrm{mg} / 100 \mathrm{~g}$ and $45.23 \mathrm{mg} / 100 \mathrm{~g}$, respectively.

Significant differences among the antioxidant activities of the various constituents measured by FRAP, HRSA, and LPIA $(P<0.01)$ were observed. In addition, the FRAPs and LPIAs of $\mathrm{C} 4, \mathrm{C} 5$, and $\mathrm{C} 6$ were higher than those of CF, and the HRSA of C6 was higher than that of CF. Similarly to the antioxidant activity, the TPCs of C4, C5, and C6 as well as the TAC of C6 were higher than those of $\mathrm{CF}$, indicating that the antioxidant activity ingredients in the chloroform fraction were further separated and concentrated through silica gel column separation. Moreover, the antioxidant activities, TPCs, and TACs of C4, C5, and C6 were high, suggesting that the correlation between the antioxidant activity of maca and the TPC as well as the TAC may be obvious.
TABLE 1: Pearson's correlation coefficients of FRAP, HRSA, and LPIA versus TPC, TAC, and TSC.

\begin{tabular}{lccc}
\hline & FRAP & HRSA & LPIA \\
\hline TPC & $0.941^{* *}$ & 0.306 & $0.639^{* *}$ \\
TAC & $0.531^{*}$ & $0.952^{* *}$ & $0.88^{* *}$ \\
TSC & $-0.477^{*}$ & -0.458 & -0.396 \\
\hline
\end{tabular}

${ }^{*}$ Correlation is significant at $P<0.05 .{ }^{* *}$ Correlation is significant at $P<$ 0.01 .

3.5. Correlation between Secondary Metabolites and Antioxidant Activity. Pearson's correlation coefficient was applied to evaluate the relationship between the antioxidant activity and secondary metabolite contents, including TPC, TAC, and TSC from C1 to C6 based on FRAP, HRSA, and LPIA, as shown in Table 1.

The TPC showed a significant positive correlation to FRAP and LPIA with Pearson's correlation coefficients of 0.941 and 0.639 , respectively, while high positive correlations between TAC versus FRAP, HRSA, and LPIA were found with Pearson's correlation coefficients of 0.531, 0.952, and 0.88 , respectively. However, negative correlations between TSC versus FRAP, HRSA, and LPIA were observed with Pearson's correlation coefficients of $-0.477,-0.458$, and -0.396 , 

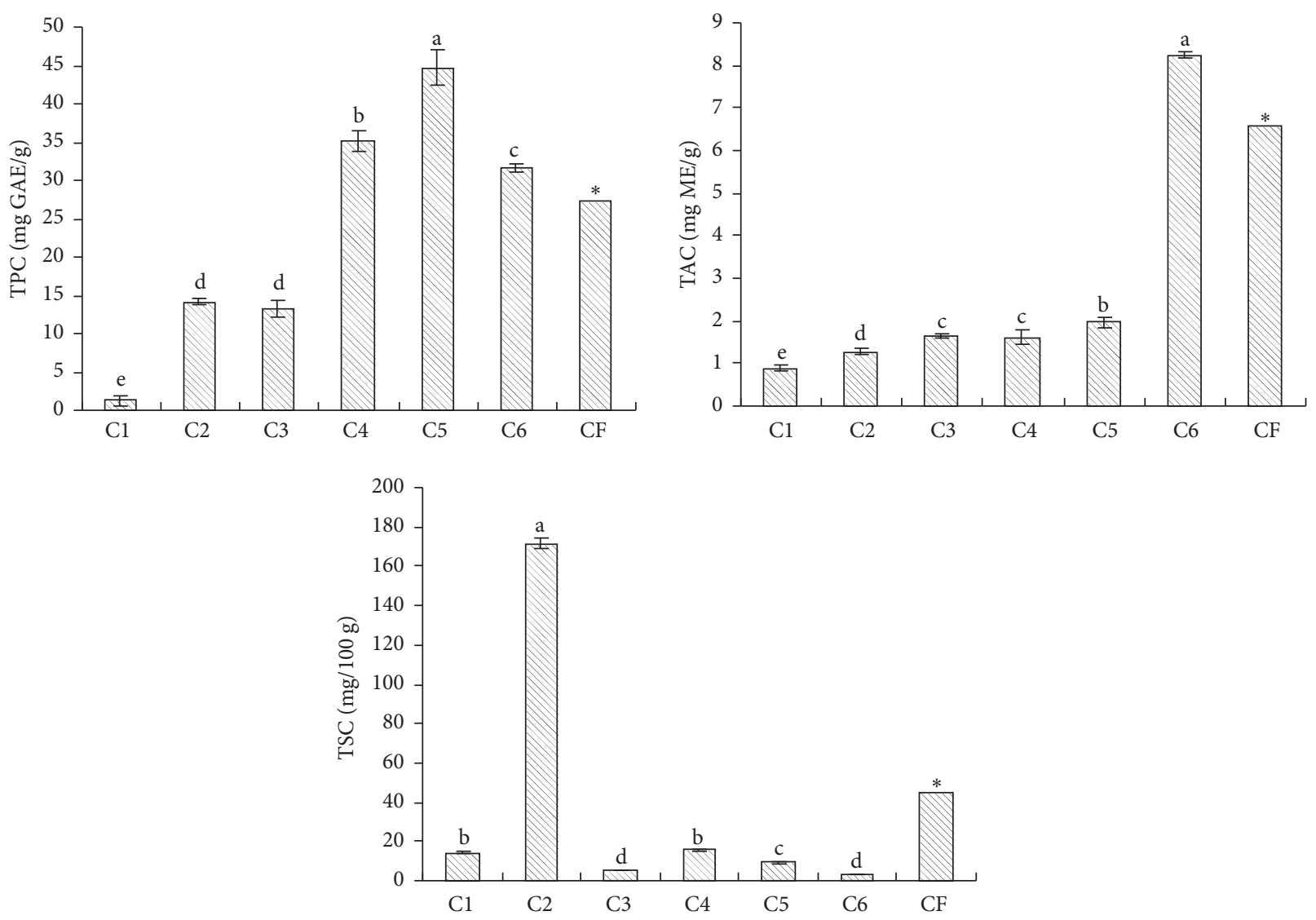

FIgURE 4: Contents of secondary metabolites of constituents separated from the chloroform fraction. The values are means \pm standard deviations of triplicate analyses. Columns labeled with different letters are significantly different $(P<0.01)$ by Duncan test. ${ }^{*}$ Values of CF are from Figure 2.

TABLE 2: Multiple linear regression models of FRAP, HRSA, and LPIA $(Y)$ with TPC and TAC $(X)$.

\begin{tabular}{|c|c|c|c|c|c|c|}
\hline Model & $\begin{array}{c}\text { Dependent } \\
\text { variable }(Y)\end{array}$ & $\begin{array}{l}\text { Independent } \\
\text { variable }(X)\end{array}$ & $\begin{array}{c}\text { Standardized } \\
\text { coefficients }\end{array}$ & Regression equation & $R^{2}$ & $\begin{array}{c}\text { Significant } \\
(P \text { value })\end{array}$ \\
\hline \multirow{2}{*}{ (1) } & \multirow{2}{*}{ FRAP } & $\operatorname{TPC}\left(X_{1}\right)$ & 0.861 & \multirow{2}{*}{$Y=4.014+1.737 X_{1}+2.688 X_{2}$} & \multirow{2}{*}{0.930} & \multirow{2}{*}{0.000} \\
\hline & & $\operatorname{TAC}\left(X_{2}\right)$ & 0.227 & & & \\
\hline$(2)$ & HRSA & $\operatorname{TAC}(X)$ & 0.952 & $Y=6.203+5.264 X$ & 0.906 & 0.000 \\
\hline \multirow[t]{2}{*}{ (3) } & \multirow[t]{2}{*}{ LPIA } & $\operatorname{TPC}\left(X_{1}\right)$ & 0.375 & \multirow{2}{*}{$Y=-13.677+0.662 X_{1}+7.742 X_{2}$} & \multirow[t]{2}{*}{0.897} & \multirow[t]{2}{*}{0.000} \\
\hline & & $\operatorname{TAC}\left(X_{2}\right)$ & 0.747 & & & \\
\hline
\end{tabular}

respectively. Moreover, the correlation coefficients between TAC versus HRSA and LPIA were higher than those between TPC versus HRSA and LPIA, suggesting that the antioxidant activity of maca was dependent on not only phenols but also alkaloids, which may be attributed to their synergistic action.

To further analyze the contributions of phenols and alkaloids to the antioxidant activity of maca as well as their synergistic effects, multiple linear regression was employed to construct multiple linear models with antioxidant activity (FRAP, HRSA, and LPIA) as the dependent variable $(Y)$ and TPC and TAC as independent variables $(X)$. Both stepwise elimination of variables (if $P>0.10$ ) and stepwise insertion of variables (if $P<0.10$ ) were performed to establish the best fit model that could exactly represent the influence of TPC and TAC on the antioxidant activity of maca.

As indicated in Table 2, model (1) was the best fit model for FRAP, indicating that FRAP was significantly linearly correlated with TPC and TAC $\left(R^{2}=0.93, P<0.05\right)$, of which $93 \%$ of FRAP variation was dependent on these factors, while the effect of TPC on FRAP was higher than that of TAC due to the standardized coefficients of TPC and TAC, 0.861 and 0.227 , respectively. The best fit model for HRSA (model (2) in Table 2) only contained TAC, suggesting that TAC (standardized coefficient $=0.952$ ) significantly linearly correlated with HRSA alone $\left(R^{2}=0.93, P<0.05\right)$ without contribution from TPC, and the $90.6 \%$ variation of HRSA 
was attributed to TAC. Similarly to FRAP, model (3) showed that LPIA was also significantly linearly correlated with both TPC and TAC $\left(R^{2}=0.897, P<0.05\right)$; thus TPC and TAC may be responsible for the $89.7 \%$ variation of LPIA. However, the effect of TAC with a standardized coefficient of 0.747 was higher than that of TPC with a standardized coefficient of 0.375 . Based on the current study, the highest maca antioxidant activity observed in the FRAP, HRSA, and LPIA assays was due to TPC and TAC, suggesting that phenols and alkaloids are the most important substances. Moreover, alkaloids seemed to have a higher correlation with antioxidation than phenols.

Both polyphenols and alkaloids are major antioxidants in natural products, and their antioxidant activities have been demonstrated in previous studies [29-31]. Several studies have shown that the antioxidant activity of maca was correlated with phenols or alkaloids; Campos et al. [32] reported that the correlation between TPC and antioxidant activity was statistically significant $(P<0.01)$ with an $R^{2}$ value of 0.68 and a correlation coefficient of 0.82 , indicating a moderately strong relationship between both variables. Wang et al. [28] found a significant correlation between TPC and antioxidant activity with an $R^{2}$ value of 0.986 . The lipid-soluble extract from maca, which has reduced serum lactate dehydrogenase and muscle lipid peroxidation in weight-loaded forced swimming rats as well as increased hepatic and muscle total glutathione, was found to contain macamides, such as N-benzylhexadecanamide and N-benzyl-5-oxo-6E,8E-octadecadienamide from a $7.8 \mathrm{mg} / \mathrm{g}$ extract, suggesting that the antioxidant effect can be partly explained by alkaloids [16]. According to $\mathrm{Du}$ [33], alkaloids extracted from maca at a concentration of $62.4 \%$ were considered the major antioxidant substance due to obvious positive correlations between alkaloids with DPPH scavenging ability and HRSA. Our study not only reinforced these previously mentioned results but also confirmed that the antioxidant activity of maca is attributed to both alkaloids and phenols by employing multiple linear regression. TAC had the most influence on antioxidant activity because its linear correlations with FRAP, HRSA, and LPIA were statistically significant with standardized coefficients of $0.227,0.952$, and 0.747 , respectively, while TPC was only linearly correlated with FRAP and LPIA with standardized coefficients of 0.861 and 0.375 , respectively. Moreover, the alkaloids in maca contained several specific compounds, such as macamides, macaenes, and macaridine that have been regarded as the primary active ingredients of maca since they were first discovered $[12,34,35]$. The antioxidant activity of maca has also been hypothesized to be the basis of other bioactivities $[9,14,17$, 18]. Thus, these specific alkaloid compounds may effectively contribute to the antioxidant activity of maca. However, the phenolic content of raw maca reported by previous studies was low, ranging from 2.5 to $9.89 \mathrm{mg} / \mathrm{g}$ [10, 16, 36-38]. The highest TPCs of maca extracts from this study and Wang et al. [28] were only 44.72 and $44.78 \mathrm{mg} / \mathrm{g}$, respectively, but the extract exhibited strong antioxidant activity with a significant correlation between the phenolic content and antioxidant activity. These results may be explained by the chemical structural differences between phenolic compounds. Phenolic compounds have radical elimination capabilities due to their abilities to donate hydrogen and form stable radical intermediates [39]. Rice-Evans et al. [40] studied the influence of the structural chemistry of polyphenols on their free radical scavenging activities. In their study, gallic acid (3,4,5-trihydroxybenzoic acid) showed higher antioxidant activity corresponding to the three available hydroxyl groups than chlorogenic acid (a glycoside of 3,4-dihydroxycinnamic acid) corresponding to the two available hydroxyl groups, which proved that the antioxidant activity of phenols depends on the number of hydroxyl groups in the molecule and chemical structure. Furthermore, the strong antioxidant activity corresponding to low TPCs may be associated with synergistic interactions between phenolic compounds and other antioxidant compounds, such as alkaloids. Based on the above results, the specific alkaloids and phenolic compounds that correlated with the antioxidant activity of maca as well as their synergistic interactions need to be further investigated.

\section{Conclusions}

In this study, the correlation between the antioxidant activity and secondary metabolites, including TPC, TAC, and TSC, from maca was investigated by FRAP, HRSA, and LPIA, in which polarity partition extraction, further separation using a silica gel column, Pearson's correlation coefficient analysis, and multiple linear regression were employed. Chloroform was an efficient solvent for extracting antioxidant compounds from maca, and the active antioxidant ingredients in the chloroform fraction were further separated and concentrated using a silica gel column. The correlation results from statistical analyses exhibited that the antioxidant activity was significantly correlated with TPC and TAC based on FRAP, HRSA, and LPIA. Moreover, TPC was significantly linearly correlated with FRAP and LPIA, while TAC was significantly linearly correlated with FRAP, HRSA, and LPIA, suggesting that the antioxidant activity of maca is not only due to phenols but also alkaloids. Thus, alkaloids and phenols are the most important substances that contribute to the antioxidation of maca, of which alkaloids seemed to have a stronger correlation with antioxidation than phenols. Further studies need to be conducted to identify alkaloids and phenolic compounds that are correlated with the antioxidant activity of maca as well as their synergistic interactions. These additional results would provide new insight into the mechanisms that lead to the special bioactivities of maca.

\section{Additional Points}

Practical Applications. Maca has gained significant interest due to its variety of bioactivities, such as fertility-enhancing properties, sexual function improvement, antifatigue, and inhibition of prostate hyperplasia. However, there is a lack of information concerning exact bioactivity compounds that correspond to a particular function. Moreover, some researchers considered that various bioactivities of maca were attributed to its antioxidant activity. Therefore the correlation between the antioxidant activity and secondary metabolites 
of maca was investigated in our study. The result showed that both phenols and alkaloids are the most important substances for the antioxidation of maca, of which the antioxidant effect of alkaloids seemed to be higher than that of phenols, and it was consistent with some reports, which regarded alkaloids in maca as primary activity ingredient. The result of present study would provide new insight into the mechanisms that lead to the special bioactivities of maca.

\section{Conflicts of Interest}

The authors declare that they have no conflicts of interest in this work.

\section{Acknowledgments}

This research was financially supported by the Special Fund Project for the Scientific Research of State Forest Public Welfare Industry, SAF, China (201004028).

\section{References}

[1] J. León, “The "Maca” (Lepidium meyenii), a little known food plant of peru," Economic Botany, vol. 18, no. 2, pp. 122-127, 1964.

[2] G. F. Gonzales, A. Ruiz, C. Gonzales, L. Villegas, and A. Cordova, "Effect of Lepidium meyenii (maca) roots on spermatogenesis of male rats," Asian Journal of Andrology, vol. 3, no. 3, pp. 231-233, 2001.

[3] A. C. Ruiz-Luna, S. Salazar, N. J. Aspajo, J. Rubio, M. Gasco, and G. F. Gonzales, "Lepidium meyenii (Maca) increases litter size in normal adult female mice," Reproductive Biology and Endocrinology, vol. 3, article 16, 2005.

[4] G. F. Gonzales, A. Córdova, K. Vega et al., "Effect of Lepidium meyenii (MACA) on sexual desire and its absent relationship with serum testosterone levels in adult healthy men," Andrologia, vol. 34, no. 6, pp. 367-372, 2002.

[5] L. J. Yu and W. W. Jin, "Study on the nutritional components and the anti-fatigue effects of dry powder of maca (Lepidium meyenii)," Food Science, vol. 25, no. 1, pp. 164-166, 2004.

[6] T. Miura, H. Motoki, Y. Naito, and I. Suzuki, "Antihypoglycemic effect of maca in fasted and insulin-induced hypoglycemic mice," Journal of Traditional Medicines, vol. 16, no. 3, pp. 93-96, 1999.

[7] Y. Z. Zhang, L. J. Yu, J. M. Wan, and W. W. Jin, "Effect of ethanol extract of Lepidium meyenii Walp. (maca) on immunological function of mice," Natural Product Research \& Development, vol. 19, no. 2, pp. 274-276, 2007.

[8] M. Gasco, L. Villegas, S. Yucra, J. Rubio, and G. F. Gonzales, "Dose-response effect of Red Maca (Lepidium meyenii) on benign prostatic hyperplasia induced by testosterone enanthate," Phytomedicine, vol. 14, no. 7-8, pp. 460-464, 2007.

[9] Y. Zhang, L. Yu, M. Ao, and W. Jin, "Effect of ethanol extract of Lepidium meyenii Walp. on osteoporosis in ovariectomized rat," Journal of Ethnopharmacology, vol. 105, no. 1-2, pp. 274-279, 2006.

[10] M. Sandoval, N. N. Okuhama, F. M. Angeles et al., "Antioxidant activity of the cruciferous vegetable Maca (Lepidium meyenii)," Food Chemistry, vol. 79, no. 2, pp. 207-213, 2002.

[11] Z. He, Y. Feng, L. F. Xu et al., "In vitro antioxidant activity of ethanol extract of maca (Lepidium meyenii walpers) cultivated in Yunnan," Food Science, vol. 31, no. 15, pp. 39-43, 2010.
[12] R. Večeřa, J. Orolin, N. Škottová et al., "The influence of maca (Lepidium meyenii) on antioxidant status, lipid and glucose metabolism in rat," Plant Foods for Human Nutrition, vol. 62, no. 2, pp. 59-63, 2007.

[13] L.-J. Yu, Y.-Z. Zhang, W.-W. Jin, Z.-Y. Ji, and W.-T. Xiong, "Antisenility effect of ethanol extract in rhizome of Lepidium meyenii in mice," Chinese Traditional and Herbal Drugs, vol. 37, no. 1, pp. 81-83, 2006.

[14] K.-J. Lee, K. Dabrowski, J. Rinchard, C. Gomez, L. Guz, and C. Vilchez, "Supplementation of maca (Lepidium meyenii) tuber meal in diets improves growth rate and survival of rainbow trout Oncorhynchus mykiss (Walbaum) alevins and juveniles," Aquaculture Research, vol. 35, no. 3, pp. 215-223, 2004.

[15] G. F. Gonzales, V. Vasquez, D. Rodriguez et al., "Effect of two different extracts of red maca in male rats with testosteroneinduced prostatic hyperplasia," Asian Journal of Andrology, vol. 9, no. 2, pp. 245-251, 2007.

[16] E. H. Choi, J. I. Kang, J. Y. Cho et al., "Supplementation of standardized lipid-soluble extract from maca (Lepidium meyenii) increases swimming endurance capacity in rats," Journal of Functional Foods, vol. 4, no. 2, pp. 568-573, 2012.

[17] C. Clément, U. Witschi, and M. Kreuzer, "The potential influence of plant-based feed supplements on sperm quantity and quality in livestock: A review," Animal Reproduction Science, vol. 132, no. 1-2, pp. 1-10, 2012.

[18] S. Yucra, M. Gasco, J. Rubio, J. Nieto, and G. F. Gonzales, "Effect of different fractions from hydroalcoholic extract of Black Maca (Lepidium meyenii) on testicular function in adult male rats," Fertility and Sterility, vol. 89, no. 5, pp. 1461-1467, 2008.

[19] I. F. F. Benzie and J. J. Strain, "The ferric reducing ability of plasma (FRAP) as a measure of 'antioxidant power': the FRAP assay," Analytical Biochemistry, vol. 239, no. 1, pp. 70-76, 1996.

[20] H. D. Xu and S. H. Qin, "Ultrasonic-assisted extraction and in vitro antioxidant activity evaluation of polysaccharides from Chaenomeles sinensis (thouin) koehne," Food Science, vol. 31, no. 10, pp. 106-111, 2010.

[21] T. B. Ng, F. Liu, and Z. T. Wang, "Antioxidative activity of natural products from plants," Life Sciences, vol. 66, no. 8, pp. 709-723, 2000.

[22] V. L. Singleton, R. Orthofer, and R. M. Lamuela-Raventós, "Analysis of total phenols and other oxidation substrates and antioxidants by means of folin-ciocalteu reagent," Methods in Enzymology, vol. 299, pp. 152-178, 1999.

[23] J. Gan, Y. Feng, Z. He, L. F. Xu, H. Zhang, and X. M. Chen, "Total alkaloids in maca (lepidium meyenii) cultivated in Yunnan," Food Science, vol. 31, no. 24, pp. 415-419, 2010.

[24] J. Gan, Y. Feng, H. Zhang, Z. He, H. Zheng, and X. Li, "Analysis on composition and content of sterols in three color types of maca, Lepidium meyenii," Journal of Forest Research, vol. 26, no. 1, pp. 129-132, 2013.

[25] M. Antolovich, P. D. Prenzler, E. Patsalides, S. McDonald, and K. Robards, "Methods for testing antioxidant activity," Analyst, vol. 127, no. 1, pp. 183-198, 2002.

[26] S. F. Sulaiman, K. L. Ooi, and Supriatno, "Antioxidant and $\alpha$ glucosidase inhibitory activities of cucurbit fruit vegetables and identification of active and major constituents from phenolicrich extracts of lagenaria siceraria and sechium edule," Journal of Agricultural and Food Chemistry, vol. 61, no. 42, pp. 1008010090, 2013.

[27] I. Orhan, M. Kartal, Q. Naz et al., "Antioxidant and anticholinesterase evaluation of selected Turkish Salvia species," Food Chemistry, vol. 103, no. 4, pp. 1247-1254, 2007. 
[28] L. W. Wang, J. Liang, X. D. Wang, X. F. Yuan, B. Zhao, and Y. W. Yang, "High efficient antioxidant activity of extracts from Lepidium meyenii Walp," Asian Journal of Chemistry, vol. 24, no. 10, pp. 4795-4798, 2012.

[29] F. Pu, X.-L. Ren, and X.-P. Zhang, "Phenolic compounds and antioxidant activity in fruits of six Diospyros kaki genotypes," European Food Research and Technology, vol. 237, no. 6, pp. 923932, 2013.

[30] B. K. Casselst, M. Asencio, P. Conget, H. Speisky, L. A. Videla, and E. A. Lissi, "Structure-antioxidative activity relationships in benzylisoquinoline alkaloids," Pharmacological Research, vol. 31, no. 2, pp. 103-107, 1995.

[31] W. Q. Yin, S. Q. Duan, Y. Zhang, L. Zeng, and X. M. Song, "Antioxidant activities of different solvents extracts and alkaloids of Uncaria rhynchophylla ( Miq.) Jacks," Journal of Guangxi Normal University, vol. 28, no. 1, pp. 31-34, 2010.

[32] D. Campos, R. Chirinos, O. Barreto, G. Noratto, and R. Pedreschi, "Optimized methodology for the simultaneous extraction of glucosinolates, phenolic compounds and antioxidant capacity from maca (Lepidium meyenii)," Industrial Crops and Products, vol. 49, pp. 747-754, 2013.

[33] G. X. Du, Study on the Isolation, Purification and Antioxidant Activities of Alkaloids in Maca, South China University of Technology, Guangzhou, China, 2011.

[34] I. Muhammad, J. Zhao, D. C. Dunbar, and I. A. Khan, "Constituents of Lepidium meyenii 'maca,' Phytochemistry, vol. 59, no. 1, pp. 105-110, 2002.

[35] B. L. Zheng, K. He, C. H. Kim et al., "Effect of a lipidic extract from Lepidium meyenii on sexual behavior in mice and rats," Urology, vol. 55, no. 4, pp. 598-602, 2000.

[36] L. G. Ranilla, Y.-I. Kwon, E. Apostolidis, and K. Shetty, "Phenolic compounds, antioxidant activity and in vitro inhibitory potential against key enzymes relevant for hyperglycemia and hypertension of commonly used medicinal plants, herbs and spices in Latin America," Bioresource Technology, vol. 101, no. 12, pp. 4676-4689, 2010.

[37] J. Rubio, S. Yucra, M. Gasco, and G. F. Gonzales, “Dose-response effect of black maca (Lepidium meyenii) in mice with memory impairment induced by ethanol," Toxicology Mechanisms and Methods, vol. 21, no. 8, pp. 628-634, 2011.

[38] J. Gan, Study on antioxidant activities and antioxidative substance basis of maca (Lepidium meyenii Walp.), Chinese Academy of Forestry, Beijing, China, 2013.

[39] W. Bors and C. Michel, "Chemistry of the antioxidant effect of polyphenols," Annals of the New York Academy of Sciences, vol. 957, pp. 57-69, 2002.

[40] C. A. Rice-Evans, N. J. Miller, and G. Paganga, "Structureantioxidant activity relationships of flavonoids and phenolic acids," Free Radical Biology \& Medicine, vol. 20, no. 7, pp. 933956, 1996. 

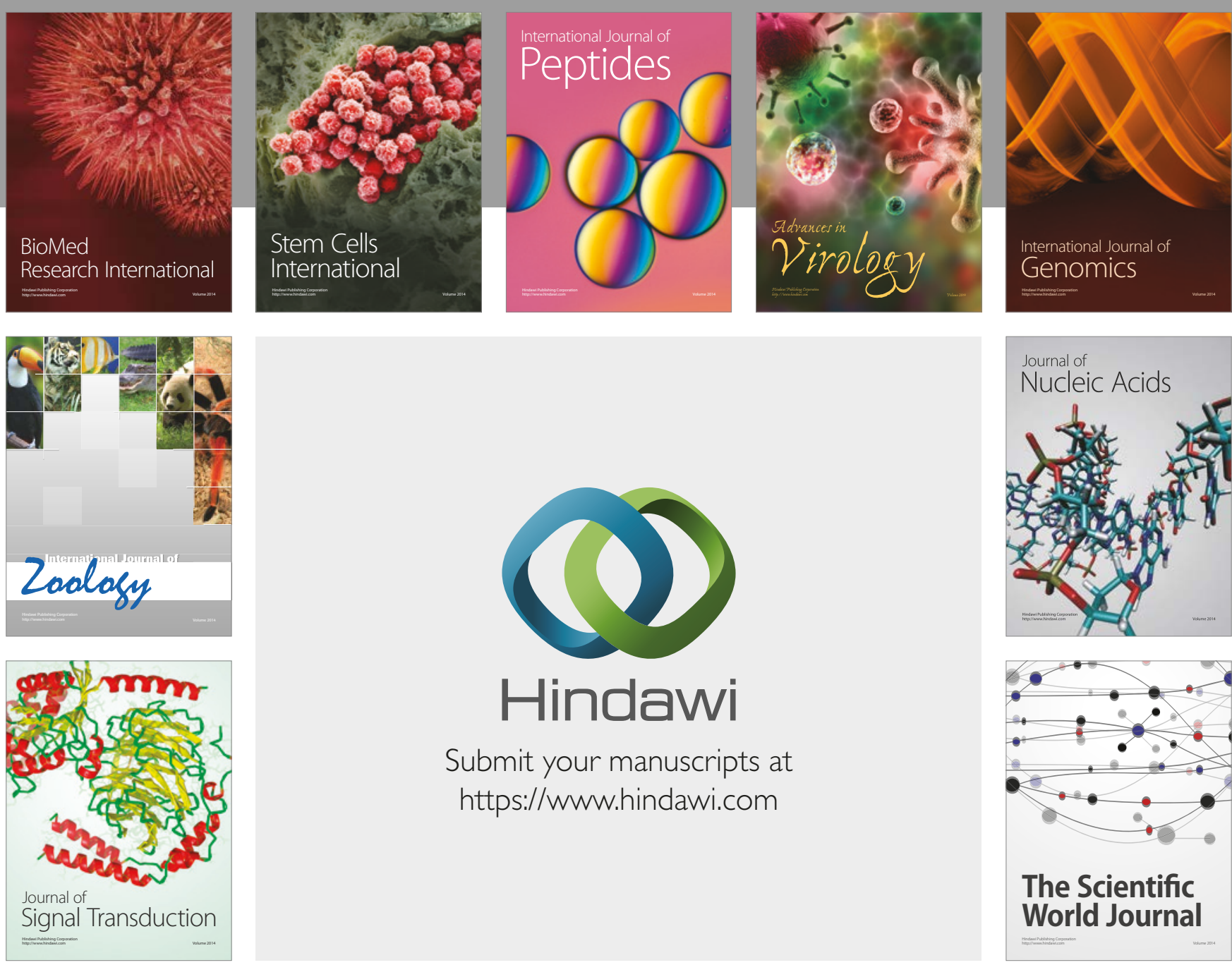

Submit your manuscripts at

https://www.hindawi.com
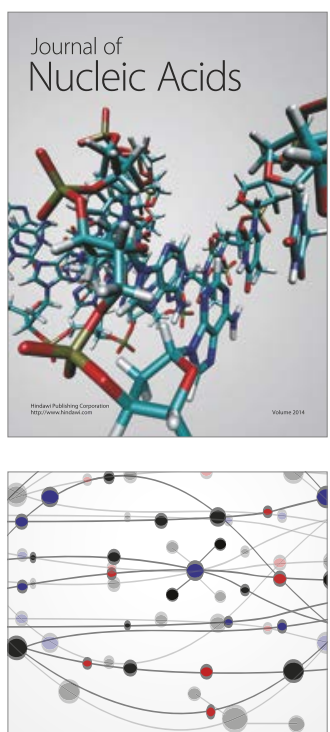

The Scientific World Journal

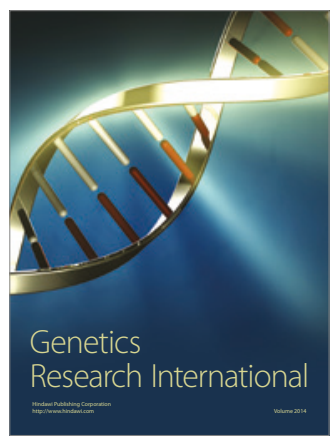

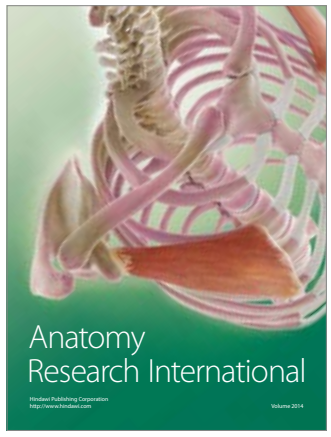

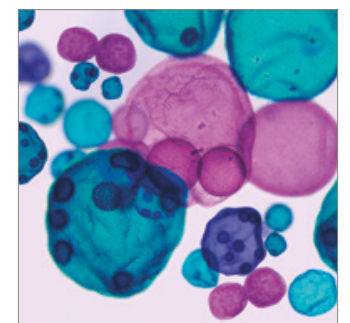

International Journal of Microbiology
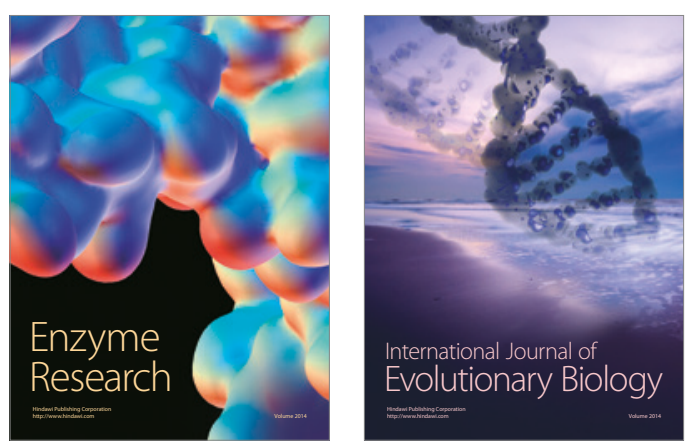
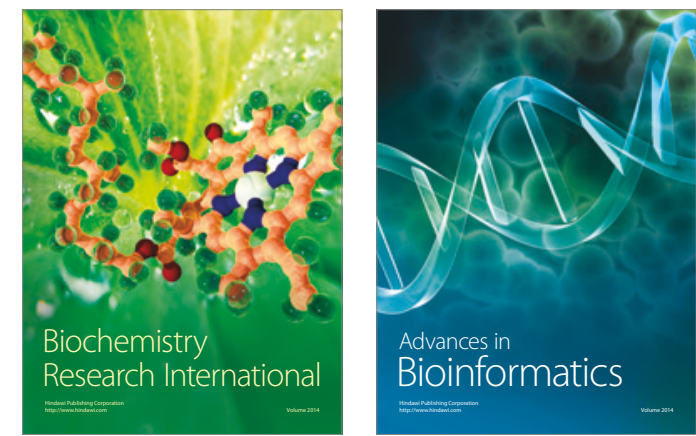

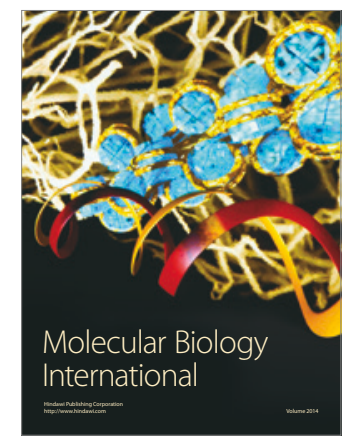

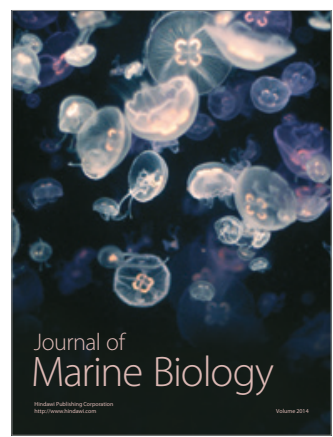

